\title{
ABC of Blood Pressure Measurement
}

\section{THE OBSERVER}

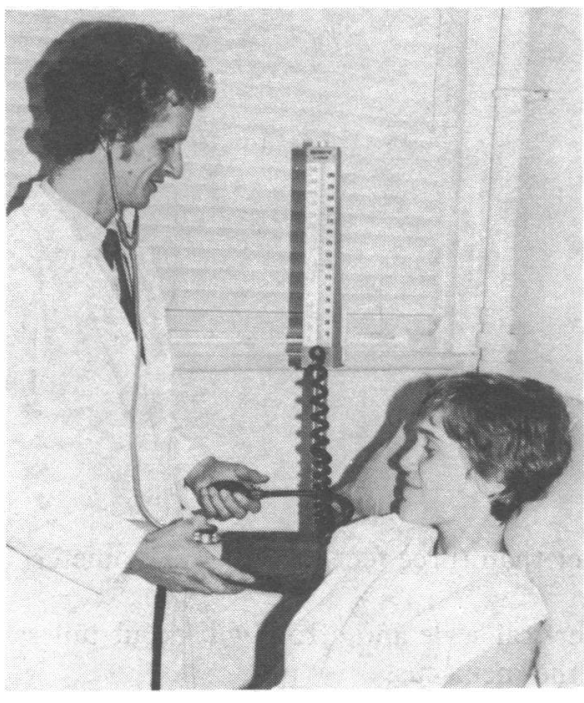

\section{Training}

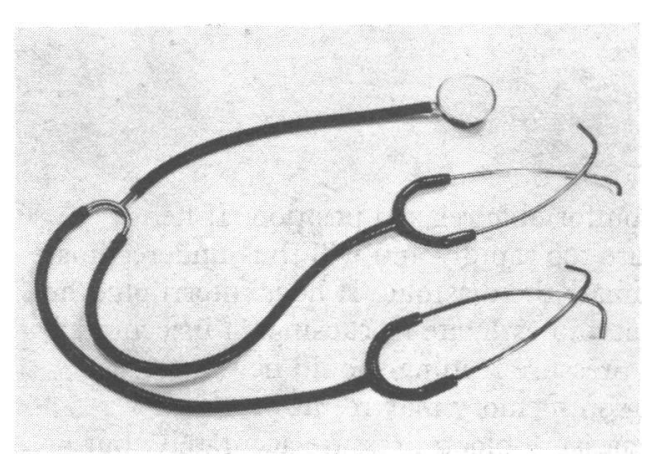

\section{What biases the observer?}

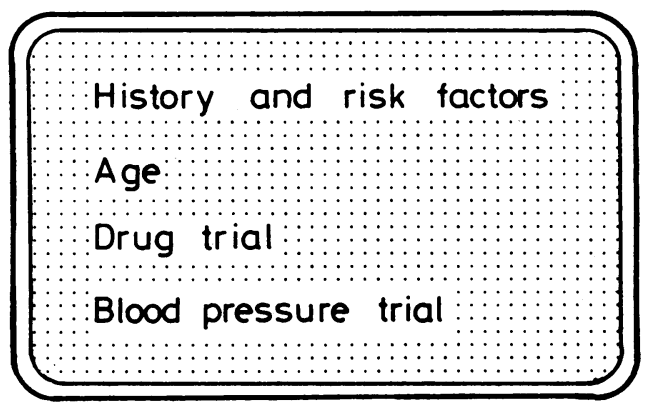

Apart from the stethoscope, the sphygmomanometer is probably used more than any other single instrument in both hospital and general practice. Doctors, nurses, and paramedical staff record blood pressure; and decisions on treatment, investigation, prognosis, fitness for insurance and employment, and epidemiological conclusions are based on these measurements. More recently patients themselves are being trained to record their own blood pressure, and there are now machines which record blood pressure in some shops and airports.

When blood pressure is measured by routine sphygmomanometry the reading is generally assumed to be accurate, and little thought is given to major errors that may arise through inadequate knowledge, carelessness, or poor maintenance of the instrument. Many doctors and nurses are unaware of potential errors of technique and fail to appreciate the limits of blood pressure measurement. They may therefore attach undue clinical importance to what is, at best, an inaccurate technique of measurement.

There is a surprising degree of variation in blood pressure interpretation among observers. Much of the variation is probably due to failure to appreciate that if indirect sphygmomanometry is to be accurate attention to detail, especially in interpreting sounds, is essential.

Nurses and medical students are often inadequately trained in blood pressure measurement. With increasing reliance on paramedical staff for recording blood pressure and the increasing interest in home recording of blood pressure much more attention needs to be directed towards training methods. Audiovisual techniques are ideally suited both to demonstrating and to assessing blood pressure measurement, and students should be introduced to the technique and given preliminary training by these means. For later training the binaural stethoscope is probably the most useful tool. It enables the trainee's general competence and visual and auditory competence to be assessed.

The observer is often unconsciously biased towards raising or lowering the patient's blood pressure. This is most likely to occur when there is an arbitrary division between normal and high blood pressure, such as $140 / 90 \mathrm{~mm} \mathrm{Hg}$. An observer might tend to record a favourable measurement in a young healthy man with a borderline increase in pressure but categorise as hypertensive an obese, middle-aged man with a similar reading. Likewise, there might be observer bias in over-reading blood pressure to include the patient in a study such as a drug trial. Adequate discussion during training to make the observer aware of this sort of bias might minimise its influence. 


\section{Terminal digit preference}

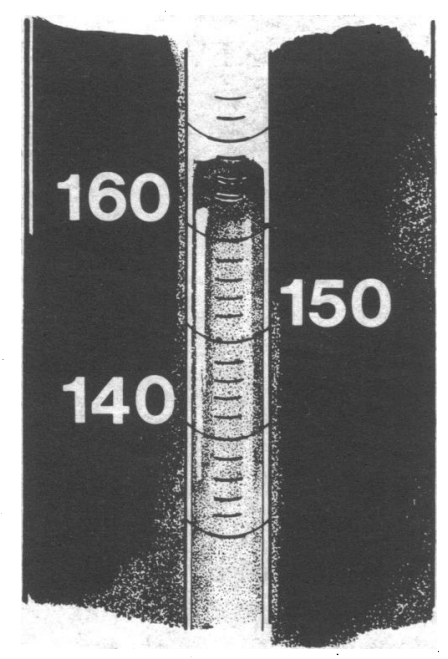

\section{Viewing distance and angle}

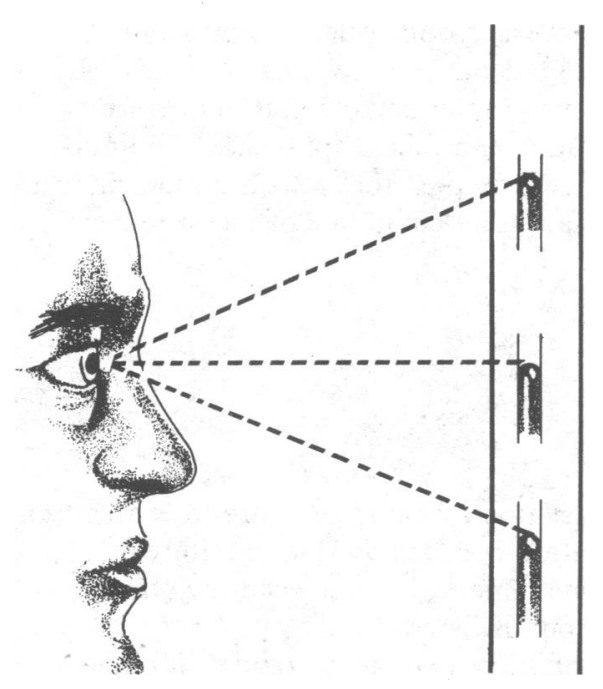

\section{Taking care and time}

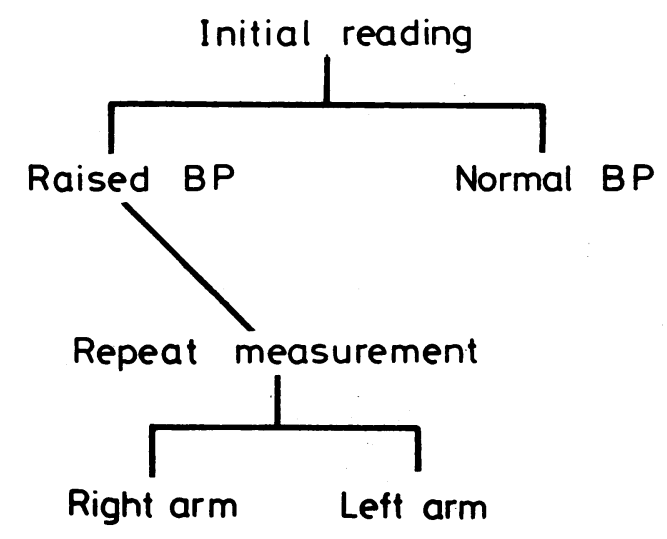

Observers show a strong preference for the terminal digits 0 and 5 , even though a $5-\mathrm{mm}$ marking does not appear on many scales. There is some evidence that careful training may minimise this source of error; and if the observer can devote enough time to recording the blood pressure and if the scale is clearly marked the pressure may be recorded to the nearest $2 \mathrm{~mm} \mathrm{Hg}$. Special sphygmomanometers have been designed to remove terminal digit preference and observer bias, but they are generally used only for research.
The observer should be no further than three feet from the manometer so that he can read the scale easily.

The mercury manometer has a vertical scale and errors will occur unless the eye is kept close to the level of the meniscus.

The aneroid scale is a composite of vertical and horizontal divisions and numbers and must be viewed straight on-with the eye on a line perpendicular to the centre of the face of the gauge.
The observer should be in a comfortable relaxed position. If he is hurried he will release the pressure too rapidly and will thus underestimate the systolic pressure and overestimate the diastolic. If he is interrupted he may forget the exact measurement and estimate it, causing an inaccurate value to be recorded. The blood pressure reading should be written as soon as measured because relying on memory may result in error.

It takes about five minutes to measure blood pressure accurately, but in practice considerably less time is devoted to the procedure. If the blood pressure is raised at the initial assessment the observer should repeat the measurement and also take the blood pressure in the other arm.

Partial loss of hearing is another potential source of error. Low-energy acoustic sounds, such as the Korotkoff sounds, may be above the normal hearing threshold when listened for with a stethoscope, and an observer may not recognise his inability to appreciate these sounds.

The Blood Pressure Evaluation and Treatment Centre, The Charitable Infirmary, Dublin: Dr Eoin O'Brien is consultant physician (cardiology) and Professor Kevin O'Malley professor of clinical pharmacology at the Royal College of Surgeons in Ireland.

This is the first of a series of seven papers, and no reprints will be available from the authors. 